\title{
An X-ray Observations of A Gradual Coronal Mass Ejections (CMEs) on 15th April 2012
}

\author{
Z. S. Hamidi ${ }^{1, *}$, N. N. M. Shariff ${ }^{2}$, C. Monstein ${ }^{3}$, W. N. A. Wan Zulkifli ${ }^{1}$, \\ M. B. Ibrahim ${ }^{1}$, N. S. Arifin ${ }^{1}$, N. A. Amran ${ }^{1}$ \\ ${ }^{1}$ School of Physics and Material Sciences, Faculty of Sciences, MARA University of Technology, \\ 40450, Shah Alam, Selangor, Malaysia \\ ${ }^{2}$ Academy of Contemporary Islamic Studies (ACIS), MARA University of Technology, \\ 40450, Shah Alam, Selangor, Malaysia \\ ${ }^{3}$ Institute of Astronomy, Wolfgang-Pauli-Strasse 27, Building HIT, Floor J, \\ $\mathrm{CH}-8093$ Zurich, Switzerland \\ *E-mail address: zetysh@salam.uitm.edu.my
}

\begin{abstract}
In the present work, we will highlight the solar observation during 15th April 2012, solar filament eruption which is accompanied by an intense and gradual Coronal Mass Ejections (CMEs) The explosion of CMEs was observed at 2:12:06 UT and also can be observed by the Solar Dynamics Observatory (SDO) with an Active Region AR1458 is crackling with C-class solar flares. The solar flare class B3 and C2 were observed beginning 2241 UT and 0142 UT. The event is considered as second largest CMEs been detected since five years. Although the solar activity within a few days is considered quite low and there are no proton events were observed at geosynchronous orbit., the is still an unexpected explosion of CMEs can be occurred. The radio flux number $(10.7 \mathrm{~cm})$ exceeds $10^{2}$ with the number of sunspot and area of sunspot increased to 77 and 270. The velocity of CMEs was calculated based on the LASCO2 data. From the results, it is clearly seen that the range of the velocity is between $200 \mathrm{kms}^{-1}$ to $2000 \mathrm{kms}^{-1}$. This wide of range proved that the mechanism of the CMEs is a gradual process. The explosion of CMEs velocity is located from $80^{\circ}-255^{\circ}$ from North of the Sun. We can then conclude that currently, the rearrangement of the magnetic field, and solar flares may result in the formation of a shock that accelerates particles ahead of the CMEs loop and an active region play an important character in this event.
\end{abstract}

Keywords: Sun Coronal Mass Ejections (CMEs); solar corona; solar flare; solar cycle

\section{INTRODUCTION}

Coronal Mass Ejections (CMEs) can be classified as a massive burst of solar wind and magnetic fields rising above the solar corona that can trigger major disturbances in Earth's magnetosphere. Since 1970s, the CMEs has been the most important manifestation of solar activity that drives the space weather near Earth [1,2]. It is also associated with a whole host of radio bursts caused by non-thermal electrons accelerated during the eruption process [3]. This event also potentially forms type II bursts originated from the solar corona, expanding as 
they climb. During this explosion, the solar plasma is heated to tens of millions of degrees, protons and electrons, with nuclei are accelerated to near the speed of light [4]. The superheated electrons from CMEs move along the magnetic field lines faster than the solar wind can flow [5]. The energetic particles are accelerated by CME-driven shocks to very great energies throughout the interplanetary medium [6]. CMEs that travel far into the interplanetary (IP) medium and affect the Earth's space environment if Earth-directed. This event takes $10^{15}-10^{16} \mathrm{~kg}$ of plasma from the low corona in the solar wind, to disturb the nearEarth space if the CME direction is favorable [7].

In principle, the CMEs can be classified into two categories, (i) flare-related CMEs and (ii) CMEs associated with filament eruption [8]. During a solar flare, CMEs also can potentially be observed. This is due to the magnetic flux in the active region. Normally, we could observe a Type III and Type II solar burst in this case [3]. Meanwhile, the filament eruption also can form the CMEs based on the evolution of the sunspot or active region behavior. The process is very complex and it is not easy to be understood. The dominant of solar bursts in this category are Type IV and Type II burst.

In the previous work, we have highlighted this event in radio region from $45-870 \mathrm{MHz}$ [9]. However, we did not explain in detailed the behavior of the energy in the x-ray region. Therefore, we make an effort to discuss in detailed because we strongly believed that the characteristics of CMEs in the X-ray region can provide a detailed finding especially at the early stage of the phenomena seems it is a short wavelength in the electromagnetic spectrum. The main objective of this paper is to discuss the domain x-ray region that associated with Coronal Mass Ejections (CMEs). We used a data from the Solar Dynamic Observatory (SDO) and GOES Satellite to explain the evolution of the explosions. Detailed on the theory of CMEs will be discussed in the next section.

\section{AN OVERVIEW OF CORONAL MASS EJECTIONS (CMES)}

In principle, there are three distinct mechanisms that are responsible for the Coronal Mass Ejections (CMEs) explosion: (i) a gradual and (ii) impulsive CMEs [10]. In general, a gradual CMEs is formed when prominences and their cavities rise up from below coronal streamers. Their leading edges accelerate gradually to speeds in the range 400-600 $\mathrm{kms}^{-1}$ before leaving $30 \mathrm{R} \odot$ [11]. In a few cases it has been possible to deduce ion abundances of the beam trapped in closed coronal loops from the broad X-ray line intensities observed. However, for impulsive CMEs, it is often associated with flares and Moreton waves, also known as a "Tsunami" wave on the visible disk of the Sun.

This fast mode wave has been reported since 1960 and an indication of the main factor of the CMEs. The explosion can travel directly across the 2-30 R $\odot$ at speeds higher than 750 $\mathrm{km} \mathrm{s}^{-1}$. This is a very dangerous situation if the event is directed the Earth [12]. The final mechanism is related to solar burst Type IV, which reflects a third stage of the flare development which is only attained for larger events and is associated with the ejection of proton flares during the explosive phase [13]. The schematic diagram of a standard solar eruption associated with CMEs is illustrated in Figure 1. 


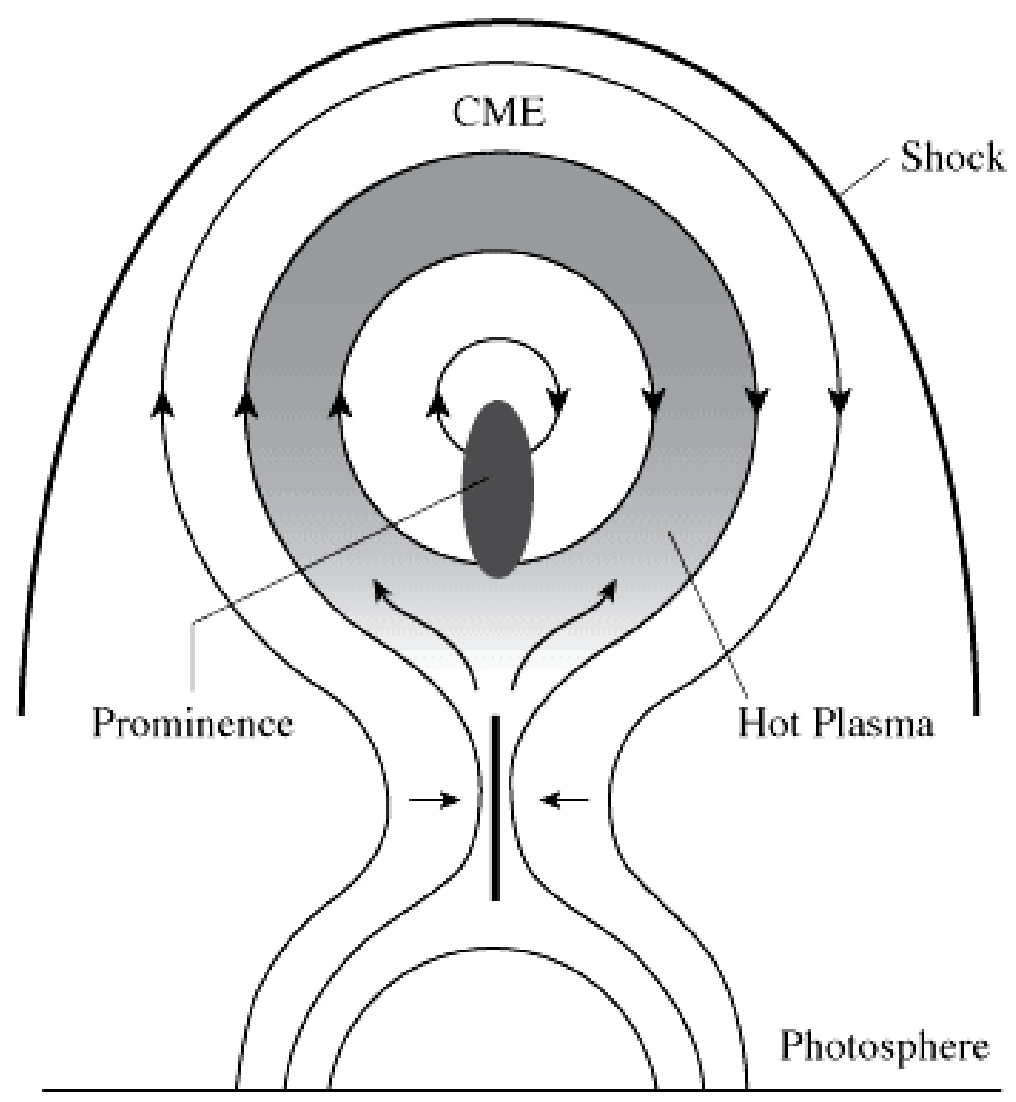

Figure 1. Schematic diagram of Coronal Mass Ejections (CMEs) explosion and the solar eruption at the photosphere of the Sun.

It is strongly believed that the solar eruption is initiated by a hot plasma, which is also categorized as an ionized state and very high temperature to accelerate the particles to eject. These particles are the moves outward beginning from the prominence point to the photosphere of the Sun. The process then produces a shock wave and can be detected as a Type II burst. In the next section, we will discuss in detailed and focus in X-ray region wavelength.

\section{RESULTS AND ANALYSIS}

The X-ray region observation has a lot of advantages in the sense of observing the solar activity. First, this short wavelength can provide a starting point of the eruption. Second, it tells the major percentage of the energy of the explosions. During 15th April 2012, solar filament eruption was accompanied by intense CMEs. In the previous work, we found that there is a Type II burst associated with this CMEs event. The observation was done at the National Space Centre Banting, Selangor with the Compound Low Cost Low Frequency Transportable Observatory (CALLISTO) system. Daily observation of the Sun from 7:00 am to $7: 00 \mathrm{pm}$ has been done since $20^{\text {th }}$ February 2012 [14-16]. Since then, we have successfully gained a lot of data and new findings of solar burst that very interesting to be discussed and highlighted [17]. We also have improved the system in term of modified the antenna to obtain a higher gain testing the antenna from time to time $[18,19]$. The Radio Frequency Interference 
(RFI) also is another important aspect that need to take into account in our observations [20]. We have chosen this event since it has been the second largest CMEs were detected since five years [21-26]. For detailed investigation, we used data obtained in an X-ray region. Figure 1 shows an explosion of CMEs observed by the Solar Dynamics Observatory (SDO) with an Active Region AR1458 is crackling with C-class solar flares. This event has also been associated with solar flare class B3, which is ejected from the corona of the Sun during 2241 UT and 0142 UT in this active region.
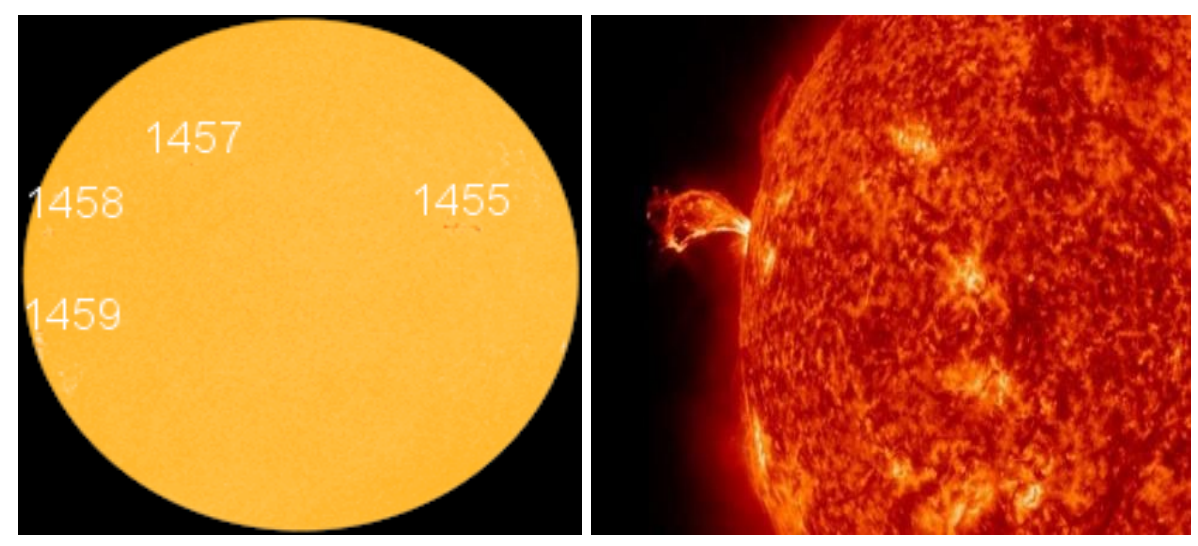

Figure 2. An Active Region of the Sun (Left) and A Coronal Mass Ejections (CMEs) from the Sun on April 15, 2012 (Right). Credit: Solar Dynamics Observatory and sunspot AR1458 is crackling with Cclass solar flares. Credit: SDO/HMI.

The explosion of CMEs was observed at 2:12:06 UT as shown in Figure 3 by LASCO2 coronagraph. The structure of helmet streamers and their instability is not easy to be understood. However, there is an indication of fast pulsations with a period of sub-second time that contributes to this event.

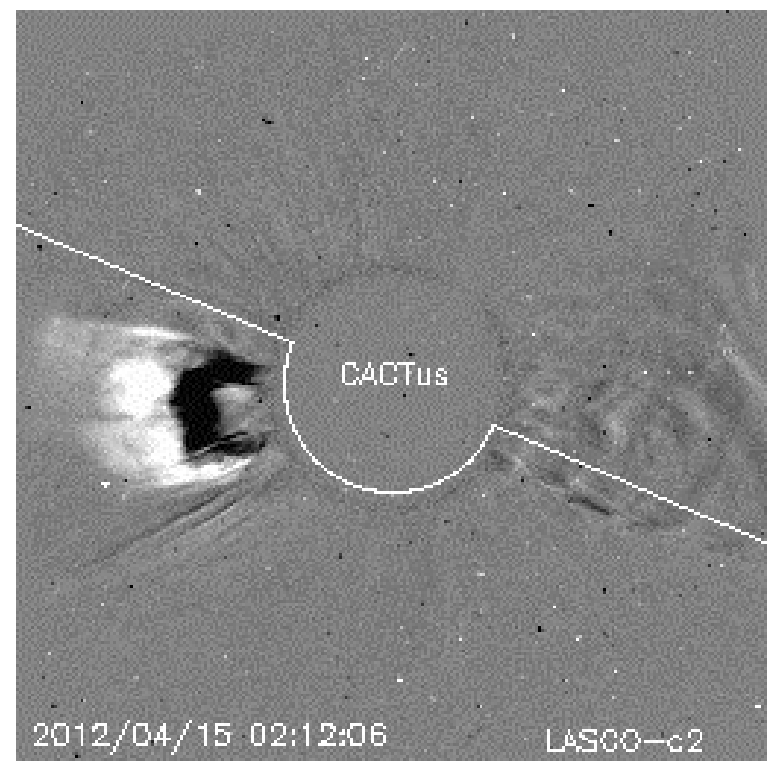

Figure 3. Coronal Mass Ejections at 2:12:06 UT (Credit: LASCO 2). 
The velocity of CMEs was calculated based on the LASCO2 data. From the Figure 4, it is clearly seen that the range of the velocity is between $200 \mathrm{kms}^{-1}$ to $2000 \mathrm{kms}^{-1}$. This wide of range proved that the mechanism of the CMEs is a gradual process. The explosion of CMEs velocity is located from $80^{\circ}-255^{\circ}$ from North of the Sun.

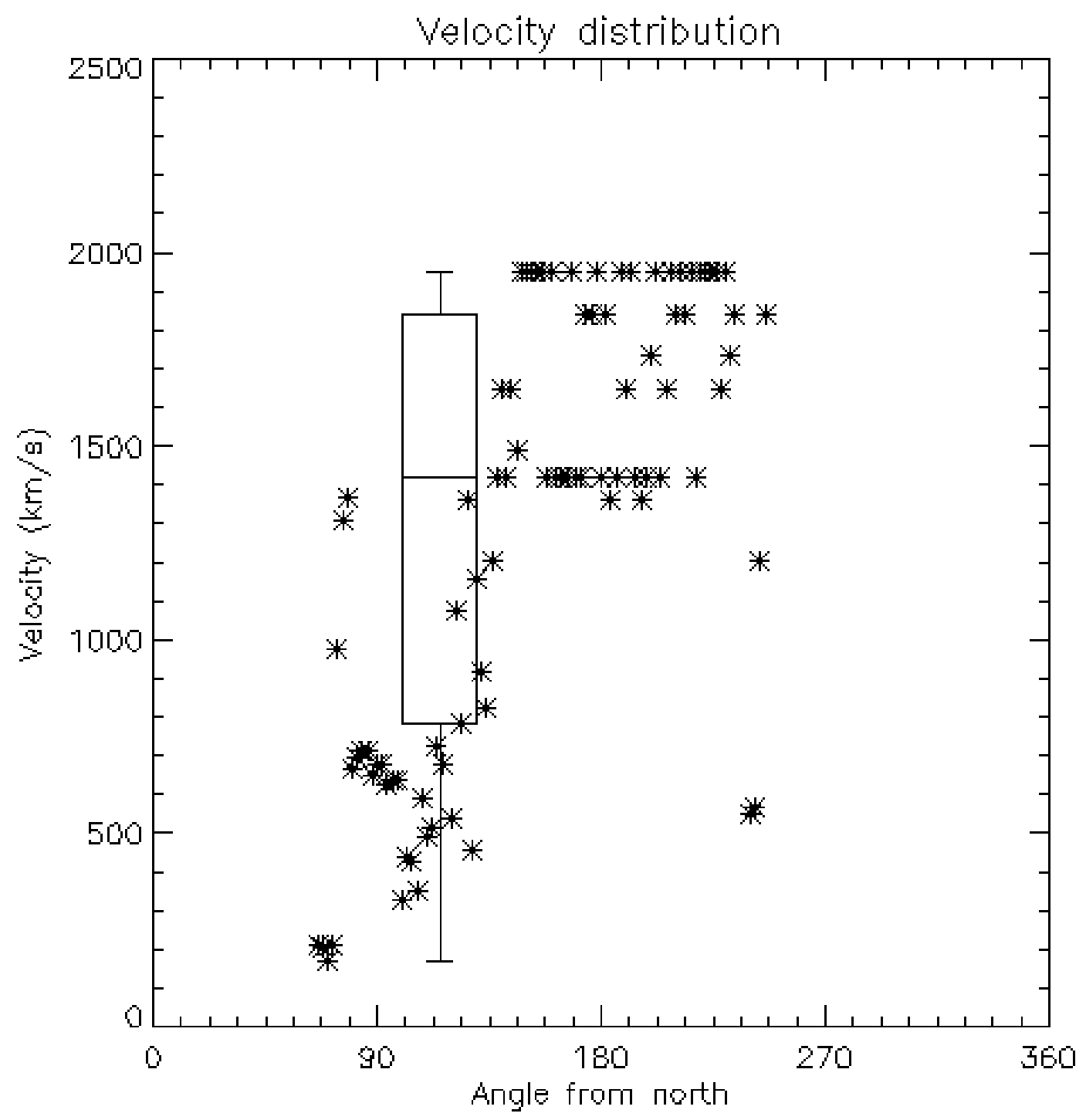

Figure 4. The range of velocity of Coronal Mass Ejection at 2:06 UT (Credit: LASCO 2).

\section{CONCLUSION}

This event since it has been the second largest CMEs were detected since five years. It should be noted that the radio flux number $(10.7 \mathrm{~cm})$ exceeds 102 with the number of sunspot and area of sunspot increased to 77 and 270 . The valuable parameters represent an active activity occur in the active region. We can then conclude that currently, the rearrangement of the magnetic field, and solar flares may result in the formation of a shock that accelerates particles ahead of the CMEs loop and an active region play an important character in this event. 


\section{ACKNOWLEDGEMENT}

We are grateful to LASCO,SDO/AIA, NOAA and SWPC make their data available online. This work was partially supported by the PPP UM PV071/2011B grants. Special thanks to C. Monstein from ETH Zurich, Switzerland who set up and gives us training on analyzing the data. Also to National Space Agency and National Space Centre for giving us a site to set up this project and support this project. Solar burst monitoring is a project of cooperation between the Institute of Astronomy, ETH Zurich, and FHNW Windisch, Switzerland, MARA University of Technology and University of Malaya. This paper also used NOAA Space Weather Prediction Centre (SWPC) for the sunspot, radio flux and solar flare data for comparison purpose. The research has made use of the National Space Centre Facility and a part of an initiative of the International Space Weather Initiative (ISWI) program.

\section{BIOGRAPHY}

Zety Sharizat Hamidi is currently a $\mathrm{PhD}$ candidate and study in Solar Astrophysics specifically in radio astrophysics at the University of Malaya. Involve a project under the International Space Weather Initiative (ISWI) and also a lecturer in School of Physics and Material Science, at MARA University of Technology, Shah Alam Selangor.

N. N. M. Shariff: Her current research is communicating sustainability. She is looking forward for cross-field research, i.e. solar astrophysics, light pollution measurement (mapping) and application of technology on sustainability.

C. Monstein is a senior Engineer at Institute of Astronomy, Wolfgang-Pauli-Strasse 27, Building HIT, Floor J, CH-8093 Zurich, Switzerland and one of the researchers who initiated the CALLISTO system around the world.

W. N. A. Wan Zulkifli, is a final year student at School of Physics and Material Science, at MARA University of Technology, Shah Alam Selangor.

M. B. Ibrahim, is a final year student at School of Physics and Material Science, at MARA University of Technology, Shah Alam Selangor.

N. S. Arifin isa final year student at School of Physics and Material Science, at MARA University of Technology, Shah Alam Selangor.

N. A. Amran is a final year student at School of Physics and Material Science, at MARA University of Technology, Shah Alam Selangor.

\section{References}

[1] J. T. Gosling, J. Geophys. Res. 98 (1993) 18937-18949.

[2] J. T. Gosling, J. Geophys. Res. 99 (1994).

[3] N. Gopalswamy, Geophysical Monograph Series 165 (2006).

[4] M. V. Goldman, D. F. Smith, Physics of the Sun, Dordrecht: Reidel, 1986.

[5] A. Kruger, Introduction to Solar Radio Astronomy and Radio Physics, D. Reidel, Publ. Co., Dordrecht, Holland, 1979.

[6] M. R. Kundu, Solar Radio Astronomy, John Wiley, 1965.

[7] W. Kundu, Solar and Space Weather Radiophysics, Dordrecht: Kluwer, 2004.

[8] N. Gopalswamy, Journal of Astrophysics and Astronomy 27 (2006) 243-254.

[9] A. O. Benz, C. Monstein, H. Meyers, CALLISTO, A New Concept for Solar Radio Spectrometer, Kluwer Academic Publishers, The Netherland, 2004.

[10] N. Gopalswamy, M. R. Kundu, Adv. Space Res. 13 (1993) 75-78. 
[11] N. Gopalswamy, et al., Sol. Phys. 259 (2009) 227-254.

[12] Z. S. Hamidi, N. Shariff, Z. Abidin, Z. Ibrahim, C. Monstein, Middle-East Journal of Scientific Research 12 (2012) 6.

[13] Z. S.Hamidi, Z. Abidin, Z. Ibrahim, C. Monstein, N. Shariff, International Journal of Fundamental Physical Sciences 2 (2012) 32-34.

[14] Z. S.Hamidi, et al., International Journal of Fundamental Physical Sciences 2 (2012) 4.

[15] Z. S. Hamidi, Z. Ibrahim, Z. Abidin, M. Maulud, N. Radzin, N. Hamzan, N. Anim, N. Shariff, International Journal of Applied Physics and Mathematics 2 (2012) 3.

[16] Z. S.Hamidi, S. Chumiran, A. Mohamad, N. Shariff, Z. Ibrahim, N. Radzin, N. Hamzan, N. Anim, A. Alias, American Journal of Modern Physics 2 (2013) 4.

[17] Z. S. Hamidi, U. F. S. U. Ibrahim, Z. Z. Abidin, Z. A. Ibrahim, N. N. M. Shariff, International Journal of Fundamental Physical Sciences 3 (2013) 20-23.

[18] Z. S.Hamidi, Z. Abidin, Z. Ibrahim, N. Shariff, C. Monstein, International Journal of Engineering Research and Development 3 (2012) 3.

[19] Z. S. Hamidi, N. N. M. Shariff, Evaluation of Signal to Noise Ratio (SNR) of Log Periodic Dipole Antenna (LPDA) BEIAC 2013, IEEE, Langkawi, Malaysia, 2013, pp. 6.

[20] Z. S.Hamidi, Z. Abidin, Z. Ibrahim, N. Shariff, Indication of radio frequency interference (RFI) sources for solar burst monitoring in Malaysia, ICPAP 2011, AIP Publisher, Indonesia, 2012, pp. 6.

[21] Z. S. Hamidi, Z. Abidin, Z. Ibrahim, N. Shariff, C. Monstein, Observations of coronal mass ejections (CMEs) at low frequency radio region on 15th April 2012, in: R. Shukor (Ed.), PERFIK 2012, American Institute of Physics, Malaysia, 2013, pp. 5.

[22] Z. S. Hamidi, N. N. M. Shariff, International Letters of Chemistry, Physics and Astronomy 5 (2014) 32-42.

[23] Z. S. Hamidi, N. N. M. Shariff, International Letters of Chemistry, Physics and Astronomy 5 (2014) 43-49.

[24] Z. S. Hamidi, N. N. M. Shariff, International Letters of Chemistry, Physics and Astronomy 7 (2014) 21-29.

[25] Z. S. Hamidi, N. N. M. Shariff, International Letters of Chemistry, Physics and Astronomy 7 (2014) 30-36.

[26] Z. S. Hamidi, N. N. M. Shariff, C. Monstein, Z. A. Ibrahim, International Letters of Chemistry, Physics and Astronomy 7 (2014) 37-44. 